\title{
An Iterative Maximum SINR Receiver for Multicarrier CDMA Systems Over a Multipath Fading Channel With Frequency Offset
}

\author{
Juinn-Horng Deng, Student Member, IEEE, and Ta-Sung Lee, Member, IEEE
}

\begin{abstract}
A robust iterative multicarrier code-division multiple-access (MC-CDMA) receiver with adaptive multipleaccess interference (MAI) suppression is proposed for a pilot symbols assisted system over a multipath fading channel with frequency offset. The design of the receiver involves a two-stage procedure. First, an adaptive filter based on the generalized sidelobe canceller (GSC) technique is constructed at each finger to perform despreading and suppression of MAI. Second, pilot symbols assisted frequency offset estimation, channel estimation and a RAKE combining give the estimate of signal symbols. In order to enhance the convergence behavior of the GSC adaptive filters, a decisions-aided scheme is proposed, in which the signal waveform is first reconstructed and then subtracted from the input data of the adaptive filters. With signal subtraction, the proposed MC-CDMA receiver can achieve nearly the performance of the ideal maximum signal-to-interference-plus noise ratio receiver assuming perfect channel and frequency offset information. Finally, a low-complexity partially adaptive (PA) realization of the GSC adaptive filters is presented as an alternative to the conventional multiuser detectors. The new PA receiver is shown to be robust to multiuser channel estimation errors and offer nearly the same performance of the fully adaptive receiver.
\end{abstract}

Index Terms-Generalized sidelobe canceller, maximum signalto-interference-plus noise ratio (MSINR) receiver, multicarrier code-division multiple access (MC-CDMA), partial adaptivity (PA).

\section{INTRODUCTION}

$\mathbf{T}$ HE DIRECT sequence code-division multiple-access (CDMA) air interface has been selected to be a major candidate for providing multimedia services in the third-generation (3G) mobile radio communications. This is mainly due to its soft multiple access characteristics, robustness against fading, and anti-interference capability. Recently, a new CDMA technology has been proposed based on the combination of CDMA and multicarrier (MC) transmission to support high data rate services [1]. The MC-CDMA systems can be categorized into two major types [1]: One is a combination of orthogonal frequency-division multiplexing (OFDM) and CDMA, and

Manuscript received August 1, 2001; revised April 8, 2002; accepted July 17,2002 . The editor coordinating the review of this paper and approving it for publication is W.-Y. Kuo. This work was supported in part by the Ministry of Education and in part by the National Science Council of R.O.C. under Contract 89-E-FA06-2-4.

The authors are with the Department of Communication Engineering and Microelectronics and Information Systems Research Center, National Chiao Tung University, Hsinchu 300, Taiwan, R.O.C. (e-mail: jhdeng.cm88g@ @ nctu.edu.tw; tslee@mail.nctu.edu.tw).

Digital Object Identifier 10.1109/TWC.2003.811188 the other is a scheme of parallel transmission of narrowband direct sequence (DS) waveforms in the frequency domain. In the former system (referred to as MC-CDMA), a spreading sequence of length $M$ is serial-to-parallel converted, and then each chip modulates a different carrier frequency. This implies that the resulting signal has a spreading sequence in the frequency domain. In the latter system (referred to as MC DS-CDMA), the available frequency spectrum is divided into $M$ equi-width frequency bands, or carriers. Each frequency band is used to transmit a narrowband DS waveform, which means that the spreading operation is done in the time domain. Both of these two types of schemes can offer frequency diversity and robustness to the adverse effect of frequency-selective fading. In addition to multipath effects, an MC-CDMA system is also subject to limiting factors such as multiple-access interference (MAI) and intercarrier interference (ICI) induced by frequency offset. In forward link synchronous transmission with good channel conditions, MAI can be eliminated by employing orthogonal spreading codes. However, this is typically not achievable in reverse link asynchronous transmission or under poor channel conditions, where orthogonality among spreading codes no longer holds.

In order to effectively combat the MAI, a CDMA receiver should be able to perform adaptive processing at the chip or symbol level [2]. On the other hand, to cope with the ICI, an OFDM receiver needs to obtain an estimate of the frequency offset before further processing [3]. Unfortunately, the frequency offset cannot be accurately estimated in the presence of strong MAI. This dictates the development of an MC-CDMA receiver with effective MAI suppression before frequency offset compensation. More importantly, the suppression of MAI must be frequency offset independent. To this end, a novel iterative adaptive receiver is proposed which involves the following implementation procedure. First, a set of adaptive filters, one for each finger, is constructed to collect multipath signals with different delays, assuming that an initial timing estimate is available. The tap weights of each adaptive filter are determined in accordance with the linearly constrained minimum variance (LCMV) [4] criterion so that strong MAI can be effectively suppressed. In particular, these LCMV adaptive filters are realized in the form of generalized sidelobe canceller (GSC) [4], and a modified blocking matrix is designed to remove the signal with frequency offset from the received data. Second, pilot symbols assisted frequency offset estimation, channel estimation and RAKE combining, together capture the resolvable 


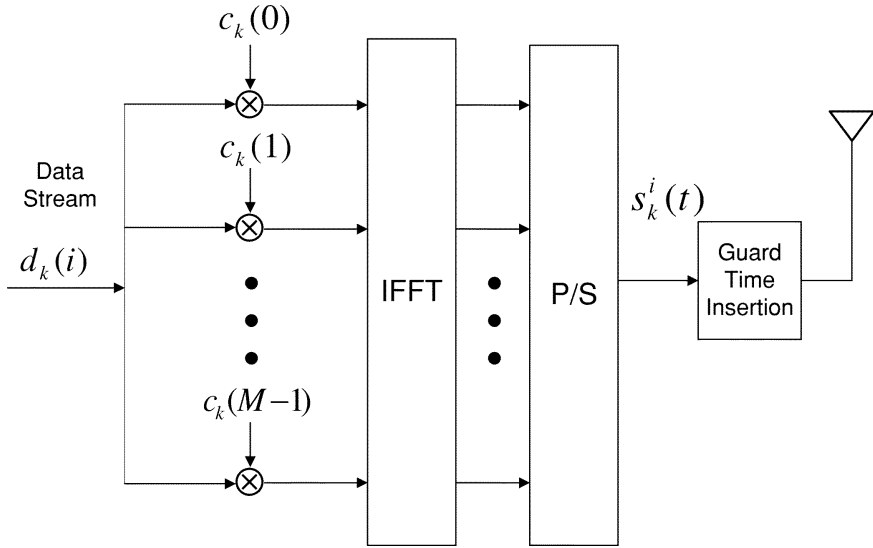

Fig. 1. Transmitter of an MC-CDMA system.

signal multipath components coherently and give the estimate of the signal symbols. In order to enhance the convergence behavior of the GSC adaptive filters, a decisions-aided scheme is proposed, in which signal waveform is first reconstructed by exploiting the frequency offset estimate, channel estimate, symbol decisions, and signal's signature. The reconstructed signal waveform is subtracted from the GSCs input data sent to the next iteration. Due to signal subtraction preprocessing, the output signal-to-interference-plus noise ratio (SINR) performance of the adaptive filters can be significantly improved [5], leading to better frequency offset estimation, channel estimation and RAKE combining, as iterations proceed. It is noteworthy that the proposed receiver subtracts the estimated signal waveform before MAI suppression. This is opposite to conventional interference cancellers [7], which estimate and subtract MAI instead. More specifically, adaptive MAI suppression is performed blindly without the signal's channel and frequency offset information. This means that the proposed receiver can be initialized and MAI can be effectively suppressed without the aid of pilot symbols and frequency offset compensation. The only information required is the timing information for performing FFT. It is shown that the proposed receiver can provide nearly the performance of the optimal maximum SINR (MSINR) receiver [5] with a few iterations. Finally, a low-complexity partially adaptive (PA) realization [8] of the GSC adaptive filters is proposed as an alternative to the conventional multiuser detectors. In particular, partial adaptivity is achieved by working with a reduced size blocking matrix obtained by projecting the estimated MAI composite channel vectors onto the range space of the original blocking matrix. It is shown that the low-complexity PA receiver is insensitive to multiuser channel estimation errors, and offers nearly the same performance of the fully adaptive receiver. In summary, the proposed MC-CDMA receiver with PA MAI suppression performs robust and near optimal signal reception with tolerance to large frequency offsets and resistance to strong MAI.

\section{MC-CDMA DATA MODEL AND MSINR RECEIVER}

Suppose that there are $K$ active users in an MC-CDMA system. Each user is assigned a unique spreading code in the

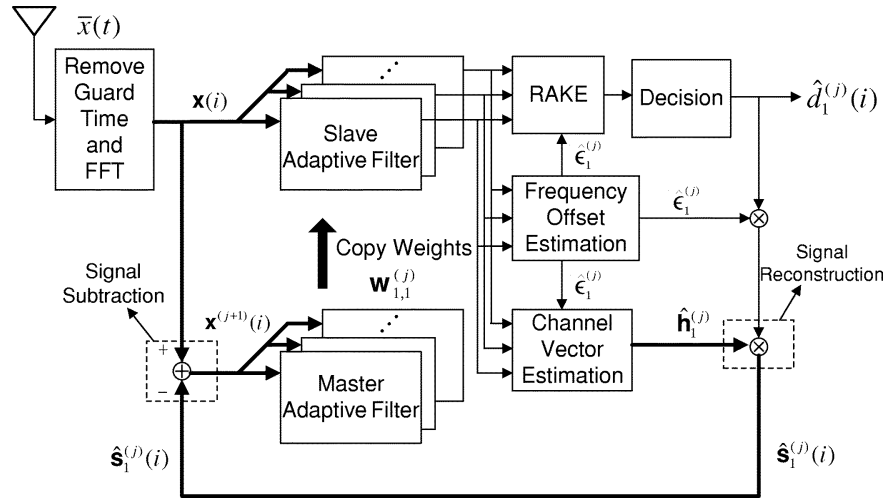

Fig. 2. Structure of proposed iterative MC-CDMA receiver.

frequency domain, as shown in Fig. 1, such that the complex baseband equivalent transmitted signal of the $k$ th user over the $i$ th data symbol can be written as [9]

$$
s_{k}^{i}(t)=\sigma_{k} \sum_{m=0}^{M-1} c_{k}(m) d_{k}(i) e^{j 2 \pi\left(m / T_{b}\right) t}
$$

where $t \in\left[i T_{b},(i+1) T_{b}\right]$, with $T_{b}$ being the symbol duration, $\sigma_{k}^{2}$ is the transmit power, $c_{k}(m)$ is the spreading code with $\left|c_{k}(m)\right|=1, d_{k}(i)$ is the $i$ th symbol assumed to be independent and identically distributed (i.i.d.) with zero-mean and unit variance and $M$ is the number of subcarriers. The transmission channel is modeled as with $L_{k}$ resolvable Rayleigh-fading paths, and a guard time of $T_{G}$ is inserted after $s_{k}^{i}(t)$ to cope with the intersymbol interference (ISI). After passing through the channel and removing the guard time, as shown in Fig. 2, the received complex baseband data can be expressed as

$$
\bar{x}(t)=\sum_{k=1}^{K} \sum_{l=1}^{L_{k}} \bar{\alpha}_{k, l} e^{j 2 \pi \Delta f_{k} t} s_{k}^{i}\left(t-\tau_{k, l}\right)+\bar{n}(t)
$$

where $\Delta f_{k}$ is the frequency offset associated with the $k$ th user (due to oscillator drifting), $\bar{\alpha}_{k, l}$ and $\tau_{k, l}$ are the complex gain and delay of the $l$ th path of the $k$ th user, respectively. $\bar{n}(t)$ is the additive white Gaussian noise (AWGN) with power $\sigma_{n}^{2}$. The received data is sampled at $t=i T_{b}+n T_{c}$ over the $i$ th symbol duration, where $T_{c}=T_{b} / M$ is the chip duration, yielding the discrete-time data samples [3]

$$
\begin{aligned}
\bar{x}_{i}(n) & =\sum_{k=1}^{K} \sum_{l=1}^{L_{k}} \bar{\alpha}_{k, l} e^{j 2 \pi \epsilon_{k}(i+(n / M))} s_{k, l}^{i}(n)+\bar{n}_{i}(n) \\
& =e^{j 2 \pi \epsilon_{1} i} \sum_{l=1}^{L_{1}} \bar{\alpha}_{1, l} e^{j 2 \pi \epsilon_{1}(n / M)} s_{1, l}^{i}(n)+\bar{u}_{i}(n)+\bar{n}_{i}(n)
\end{aligned}
$$

for $n=0,1, \ldots, M-1$, where user 1 is assumed the desired user

$$
\bar{u}_{i}(n)=\sum_{k=2}^{K} e^{j 2 \pi \epsilon_{k} i} \sum_{l=1}^{L_{k}} \bar{\alpha}_{k, l} e^{j 2 \pi \epsilon_{k}(n / M)} s_{k, l}^{i}(n)
$$

is the MAI, $s_{k, l}^{i}(n)=\left.s_{k}^{i}\left(t-\tau_{k, l}\right)\right|_{t=i T_{b}+n T_{c}}$, and $\epsilon_{k}=\Delta f_{k} T_{b}$ is the normalized frequency offset, $k=1,2, \ldots, K$. 
After fast Fourier transform (FFT), the received data in the frequency domain is given by

$$
\begin{aligned}
x_{i}(m)= & \sum_{n=0}^{M-1} \bar{x}_{i}(n) e^{-j 2 \pi(m / M) n} \\
= & e^{j 2 \pi \epsilon_{1} i}\left\{\sum_{l=1}^{L_{1}} \alpha_{1, l} c_{1, l}(m)+v_{1}(m)\right\} d_{1}(i) \\
& +u_{i}(m)+n_{i}(m)
\end{aligned}
$$

for $m=0,1, \ldots, M-1$, where

$$
\alpha_{1, l}=\sigma_{1} \bar{\alpha}_{1, l} \frac{\sin \left(\pi \epsilon_{1}\right)}{\sin \left(\pi \epsilon_{1} / M\right)} e^{j \pi \epsilon_{1}((M-1) / M)}
$$

is the equivalent complex path gain with the effects of transmit power and frequency offset incorporated

$$
c_{1, l}(m)=c_{1}(m) e^{-j 2 \pi(m / M)\left(\tau_{1, l} / T_{c}\right)}
$$

is the spreading code sequence premultiplied by the linear phase due to path delay $\tau_{1, l}$, and $u_{i}(m)$ and $n_{i}(m)$ are the FFT of $\bar{u}_{i}(n)$ and $\bar{n}_{i}(n)$, respectively. The term $v_{1}(m)$ is the ICI at the $m$ th subcarrier induced by the frequency offset and is given by

$$
v_{1}(m)=\sum_{l=1}^{L_{1}} \sum_{p=0, p \neq m}^{M-1} \beta_{1, l}\left(m, p, \epsilon_{1}\right) c_{1, l}(p)
$$

with

$$
\begin{aligned}
& \beta_{1, l}\left(m, p, \epsilon_{1}\right)=\sigma_{1} \bar{\alpha}_{1, l} \frac{\sin \left(\pi\left[\epsilon_{1}-(m-p)\right]\right)}{\sin \left(\pi\left[\epsilon_{1}-(m-p)\right] / M\right)} \\
& \times e^{j \pi\left(\epsilon_{1}-(m-p)\right)((M-1) / M)}
\end{aligned}
$$

being the corresponding complex gains. Note that the first component on the righthand side of (5) is the $i$ th symbol $d_{1}(i)$ premultiplied by an effective channel gain due to multipath fading and frequency offset.

The post-FFT received data over the $i$ th symbol can be put into the $M \times 1$ vector

$$
\begin{aligned}
\mathbf{x}(i) & =\left[x_{i}(0), x_{i}(1), \ldots, x_{i}(M-1)\right]^{T} \\
& =e^{j 2 \pi \epsilon_{1} i}\left\{\sum_{l=1}^{L_{1}} \alpha_{1, l} \mathbf{c}_{1, l}+\mathbf{v}_{1}\right\} d_{1}(i)+\mathbf{i}(i)+\mathbf{n}(i)
\end{aligned}
$$

where

$$
\begin{aligned}
\mathbf{c}_{1, l}= & {\left[c_{1, l}(0), c_{1, l}(1), \ldots, c_{1, l}(M-1)\right]^{T} } \\
= & {\left[c_{1}(0), c_{1}(1) e^{-j 2 \pi(1 / M)\left(\tau_{1}, l / T_{c}\right)}, \ldots,\right.} \\
& \left.c_{1}(M-1) e^{-j 2 \pi((M-1) / M)\left(\tau_{1, l} / T_{c}\right)}\right]^{T}
\end{aligned}
$$

is the post-FFT signature vector associated with the lth path of user 1

$$
\begin{aligned}
\mathbf{v}_{1} & =\left[v_{1}(0), v_{1}(1), \ldots, v_{1}(M-1)\right]^{T} \\
& =\sum_{l=1}^{L_{1}} \sum_{p=0, p \neq m}^{M-1} \mathbf{b}_{1, l}\left(m, p, \epsilon_{1}\right) c_{1, l}(p)
\end{aligned}
$$

is the ICI vector, $\mathbf{b}_{1, l}\left(m, p, \epsilon_{1}\right)=\left[\beta_{1, l}\left(0, p, \epsilon_{1}\right), \ldots\right.$, $\left.\beta_{1, l}\left(M-1, p, \epsilon_{1}\right)\right]^{T}, \mathbf{i}(i)=\left[u_{i}(0), \ldots, u_{i}(M-1)\right]^{T}$ is the
MAI vector, $\mathbf{n}(i)=\left[n_{i}(0), \ldots, n_{i}(M-1)\right]^{T}$ is the noise vector, and $T$ denotes the transpose. Rewriting (10), we get

$$
\mathbf{x}(i)=\mathbf{h}_{1} d_{1, \epsilon}(i)+\mathbf{i}(i)+\mathbf{n}(i)=\mathbf{s}_{1}(i)+\mathbf{i}(i)+\mathbf{n}(i)
$$

where

$$
\mathbf{h}_{1}=\sum_{l=1}^{L_{1}} \alpha_{1, l} \mathbf{c}_{1, l}+\mathbf{v}_{1}=E\left\{\mathbf{x}(i) d_{1, \epsilon}^{*}(i)\right\}
$$

is the equivalent composite channel vector of user 1 due to frequency offset and

$$
d_{1, \epsilon}(i)=d_{1}(i) e^{j 2 \pi \epsilon_{1} i}
$$

is the "frequency shifted" symbol varying with time index $i$, and $\mathbf{s}_{1}(i)=\mathbf{h}_{1} d_{1, \epsilon}(i)$ is the signal vector. With the data model in (13), it is clear that a receiver for user 1 is one that identifies and removes $\mathbf{h}_{1}$ to retrieve $d_{1, \epsilon}(i)$. Frequency offset compensation then recovers $d_{1}(i)$ from $d_{1, \epsilon}(i)$. For example, a linear receiver combines the entries of $\mathbf{x}(i)$ into an estimate of $d_{1, \epsilon}(i)$, i.e.,

$$
\hat{d}_{1, \epsilon}(i)=\mathbf{w}_{1}^{H} \mathbf{x}(i)
$$

where $\mathbf{w}_{1}$ is the weight vector, and $H$ denotes the conjugate transpose. The weight vector can be chosen in accordance with the popular minimum mean-square error (MMSE) or MSINR criterion [5]. For the MMSE receiver, we have

$$
\mathbf{w}_{1}=\mathbf{R}_{x}^{-1} \mathbf{h}_{1}
$$

where

$$
\mathbf{R}_{x}=E\left\{\mathbf{x}(i) \mathbf{x}^{H}(i)\right\}=\mathbf{h}_{1} \mathbf{h}_{1}^{H}+\mathbf{R}_{i n}
$$

is the post-FFT data correlation matrix, with

$$
\mathbf{R}_{i n}=E\left\{(\mathbf{i}(i)+\mathbf{n}(i))(\mathbf{i}(i)+\mathbf{n}(i))^{H}\right\}
$$

being the post-FFT interference-plus-noise correlation matrix. For the MSINR receiver, we have

$$
\mathbf{w}_{1}=\mathbf{R}_{i n}^{-1} \mathbf{h}_{1} \text {. }
$$

Since the channel vector $\mathbf{h}_{1}$ is obtained with the frequency shifted training symbols, the receivers in (17) and (20) are referred to as the frequency shifted (FS) MMSE and MSINR receivers, respectively.

Finally, from (13) and (16), with the frequency offset $\epsilon_{1}$ estimated as $\hat{\epsilon}_{1}$, the symbol decision $\hat{d}_{1}(i)$ can be obtained by

$$
\hat{d}_{1}(i)=\operatorname{dec}\left\{\hat{d}_{1, \epsilon}(i) e^{-j 2 \pi \hat{\epsilon}_{1} i}\right\}
$$

Popular frequency offset estimators are readily found in the literature [3] and [10]. For example, the maximum-likelihood estimator (MLE) [3] is the optimum estimator under AWGN

$$
\hat{\epsilon}_{1}=\frac{1}{2 \pi} \tan ^{-1}\left\{\frac{\sum_{i=1}^{N_{p}-1} \operatorname{Im}\left[\hat{d}_{1, \epsilon}(i+1) d_{1}^{*}(i+1) \hat{d}_{1, \epsilon}^{*}(i) d_{1}(i)\right]}{\sum_{i=1}^{N_{p}-1} \operatorname{Re}\left[\hat{d}_{1, \epsilon}(i+1) d_{1}^{*}(i+1) \hat{d}_{1, \epsilon}^{*}(i) d_{1}(i)\right]}\right\}
$$


where $N_{p}$ is the number of pilot symbols $\left\{d_{1}(i)\right\}$. Most existing frequency offset estimators are developed based on the assumption of no or little interference (i.e., AWGN). With the presence of strong MAI, these estimators often degrade seriously. It is, thus, necessary to perform interference suppression before frequency offset estimation. In the next section, an iterative adaptive receiver will be proposed which initializes without the aid of channel and frequency offset estimation, and approaches the optimal MSINR receiver in an successive fashion via signal reconstruction and subtraction.

\section{DeVelopment of Proposed MC-CDMA ReCEIVER}

An iterative MC-CDMA receiver is developed whose overall schematic diagram is depicted in Fig. 2. The receiver consists of an upper branch and a lower branch. The upper branch consists of the "slave" adaptive filter bank, frequency offset estimator, composite channel vector estimator, and RAKE combiner. The lower branch involves the "master" adaptive filter bank and signal reconstruction. At the $j$ th iteration, the master adaptive filters process the "signal subtracted" data $\mathbf{x}^{(j)}(i)$ and compute an adaptive weight vector for each finger, which provides effective signal (including multipath and intercarrier interference) reception and MAI suppression. The weight vectors of the master adaptive filters are then copied to the slave adaptive filters, which operate on the original data $\mathbf{x}(i)$ to yield signal symbol decisions. In summary, the design of the receiver involves the following procedure. First, a slave adaptive filter for each finger transforms $\mathbf{x}(i)$ from the chip domain to symbol domain and performs MAI suppression. Second, frequency offset estimation $\left(\hat{\epsilon}_{1}^{(j)}\right)$, path gain estimation $\left(\hat{\alpha}_{1, l}^{(j)} \mathrm{s}\right)$, and a RAKE combiner give the signal symbol decisions $\left(\hat{d}_{1}^{(j)}(i)\right)$. Finally, composite channel vector estimation $\left(\hat{\mathbf{h}}_{1}^{(j)}\right)$ and waveform reconstruction $\left(\hat{\mathbf{s}}_{1}^{(j)}(i)\right)$ is done by exploiting the frequency offset estimate, path gain estimate, signal symbol decisions, and signal's signature vectors. The reconstructed signal waveform is then subtracted from the data sent to the next iteration to obtain an estimate of $\mathbf{R}_{\text {in }}$ for the master adaptive filter bank. It is noteworthy that the master and slave adaptive filters use different input data.

\section{A. Construction of GSC-Based Master Adaptive Filters}

The following development considers the design of the master adaptive filter bank in the lower branch. Suppose that at the $j$ th iteration, the RAKE combiner consists of $L_{1}$ fingers, with each finger equipped with an adaptive filter. The adaptive filter at the $l$ th finger is a linear combiner matched to the post-FFT signature vector $\mathbf{c}_{1, l}$ whose output is given by

$$
y_{1, l}^{(j)}(i)=\mathbf{w}_{1, l}^{(j)^{H}} \mathbf{x}^{(j)}(i)
$$

where $\mathbf{w}_{1, l}^{(j)}$ is the weight vector, and $\mathbf{x}^{(j)}(i)$ is the post-FFT data vector. Note that, as depicted in Fig. $2, \mathbf{x}^{(1)}(i)=\mathbf{x}(i)$. To ensure an effective suppression of MAI, adaptive cancellation is performed for each of the $L_{1}$ filters. A popular criterion for

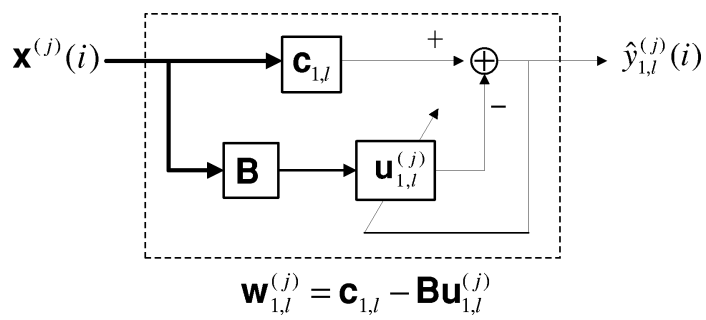

Fig. 3. Structure of GSC for master adaptive filters.

interference cancellation is the LCMV criterion [4] which determines the weight vectors according to

$$
\min _{\mathbf{w}_{1, l}^{(j)}} \mathbf{w}_{1, l}^{(j)}{ }^{H} \mathbf{R}_{x}^{(j)} \mathbf{w}_{1, l}^{(j)} \quad \text { subject to: } \mathbf{w}_{1, l}^{(j)}{ }^{H} \mathbf{c}_{1, l}=1
$$

for $l=1, \ldots, L_{1}$, where

$$
\mathbf{R}_{x}^{(j)}=E\left\{\mathbf{x}^{(j)}(i) \mathbf{x}^{(j)}{ }^{H}(i)\right\}
$$

is the post-FFT data correlation matrix at the $j$ th iteration.

In LCMV combining, the adverse phenomenon of signal cancellation usually occurs due to the mismatch of signature vectors [11]. With such mismatch present, the signal can be treated as interference and receive a very small gain. An effective solution proposed herein is to employ the scheme of GSC [4], which is essentially an indirect but simpler implementation of the LCMV algorithm. The concept of GSC, as depicted in Fig. 3, is to decompose the weight vector into two branches: $\mathbf{w}_{1, l}^{(j)}=$ $\mathbf{c}_{1, l}-\mathbf{B} \mathbf{u}_{1, l}^{(j)}$. In the upper branch, $\mathbf{c}_{1, l}$ is the fixed matched filter. In the lower branch, $\mathbf{B}$ is a predesigned "blocking" matrix which removes the signal component (including multipath and intercarrier interference) in $\mathbf{x}^{(j)}(i)$. Thus, the upper branch contains both the signal and MAI, and the lower branch contains only the MAI. The goal is then to choose the adaptive weight vector $\mathbf{u}_{1, l}^{(j)}$ to cancel the MAI in the upper branch output. To apply the GSC in constructing the adaptive filter bank, two modifications should be made. First, instead of blocking signals for a specific path, $\mathbf{B}$ must remove signals from the entire delay spread so as to avoid signal cancellation. Second, since $\mathbf{B}$ is required to remove all multipath signals, it is natural to share the same $\mathbf{B}$ for all $L_{1}$ fingers, instead of using a different matrix for each adaptive filter. Following the procedure of GSC, $\mathbf{u}_{1, l}^{(j)}$ is determined by the following MMSE problem:

$$
\min _{\mathbf{u}_{1, l}^{(j)}} E\left\{\left|\mathbf{c}_{1, l}^{H} \mathbf{x}^{(j)}(i)-\mathbf{u}_{1, l}^{(j)}{ }^{H} \mathbf{B}^{H} \mathbf{x}^{(j)}(i)\right|^{2}\right\} .
$$

Solving for $\mathbf{u}_{1, l}^{(j)}$ and substituting in $\mathbf{w}_{1, l}^{(j)}=\mathbf{c}_{1, l}-\mathbf{B} \mathbf{u}_{1, l}^{(j)}$ gives

$$
\mathbf{w}_{1, l}^{(j)}=\left[\mathbf{I}-\mathbf{B}\left(\mathbf{B}^{H} \mathbf{R}_{x}^{(j)} \mathbf{B}\right)^{-1} \mathbf{B}^{H} \mathbf{R}_{x}^{(j)}\right] \mathbf{c}_{1, l}
$$

for $l=1, \ldots, L_{1}$. Since the upper branch contains both the signal and MAI, and the lower branch contains the MAI only, minimizing the error between the two branches leads naturally to an adaptive weight vector that cancels the MAI. The solution in (27) involves the inversion of $\mathbf{B}^{H} \mathbf{R}_{x}^{(j)} \mathbf{B}$ whose size depends on the number of columns of $\mathbf{B}$. In the following sections, methods for choosing $\mathbf{B}$ and efficient realization will be discussed. 


\section{B. Selection of Blocking Matrices}

As seen in (13), the blocking matrix B should satisfy $\mathbf{B}^{H} \mathbf{h}_{1}=\mathbf{0}$ in order to remove the signal. Unfortunately, $\mathbf{h}_{1}$ is not available, and a feasible alternative approach would be to remove the possible components constituting $\mathbf{h}_{1}$. Ignoring the frequency offset, $\mathbf{B}$ can be chosen to be a full rank $M \times\left(M-L_{1}\right)$ matrix whose columns are orthogonal to $\left\{\mathbf{c}_{1,1}, \mathbf{c}_{1,2}, \ldots, \mathbf{c}_{1, L_{1}}\right\}$, as suggested by (14). With the unknown frequency offset taken into account, $\mathbf{B}$ should be constructed to remove signal components within a "broad" range of hypothesized frequency offset values. To this end, a method is proposed in which a dense set of frequencies $f_{n}, n=1,2, \ldots, N_{f}$, is chosen to accommodate an offset range $\Delta F_{s}<1 / T_{b}$, and $\mathbf{B}$ is chosen to be an $M \times M^{\prime}$ matrix whose columns are orthogonal to $\left\{\mathbf{c}_{1,1}\left(f_{n}\right), \mathbf{c}_{1,2}\left(f_{n}\right), \ldots, \mathbf{c}_{1, L_{1}}\left(f_{n}\right)\right\}, n=1,2, \ldots, N_{f}$, where

$$
\begin{aligned}
\mathbf{c}_{1, l}\left(f_{n}\right)=\operatorname{FFT}\{ & \operatorname{IFFT}\left\{\mathbf{c}_{1, l}\right\} \\
& \left.\odot\left[1, e^{j 2 \pi f_{n}}, \ldots, e^{j 2 \pi(M-1) f_{n}}\right]^{T}\right\}
\end{aligned}
$$

is the distorted post-FFT signature vector of the $l$ th path due to frequency offset $f_{n}$, with $\odot$ denoting the Hadamard (elementwise) product. Depending on the width of $\Delta F_{s}$, the above $L_{1} N_{f}$ signature vectors will span an effective rank of $M-M^{\prime}$. In other words, a set of $M-M^{\prime}$ vectors can be found well representing these signature vectors. A simple method for finding these representing vectors is via the eigenvalue decomposition of the following matrix:

$$
\mathbf{R}_{f}=\frac{1}{L_{1} N_{f}} \sum_{l=1}^{L_{1}} \sum_{n=1}^{N_{f}} \mathbf{c}_{1, l}\left(f_{n}\right) \mathbf{c}_{1, l}^{H}\left(f_{n}\right)=\sum_{i=1}^{M} \lambda_{i} \mathbf{e}_{i} \mathbf{e}_{i}^{H}
$$

where $\left\{\lambda_{i}\right\}$ and $\left\{\mathbf{e}_{i}\right\}$ are the eigenvalues and eigenvectors of $\mathbf{R}_{f}$ in descending order. Depending on the width of $\Delta F_{s}$, there will be $M-M^{\prime}$ dominant modes (with large eigenvalues) in (29). The blocking matrix $\mathbf{B}$ can then be chosen to consist of the remaining $M^{\prime}$ eigenvectors associated with the smaller eigenvalues

$$
\mathbf{B}=\left\{\mathbf{e}_{M-M^{\prime}+1}, \mathbf{e}_{M-M^{\prime}+2}, \ldots, \mathbf{e}_{M}\right\} .
$$

Due to the orthogonality among $\mathbf{e}_{i} \mathrm{~s}$, the so constructed $\mathbf{B}$ should satisfy $\mathbf{B}^{H} \mathbf{c}_{1, l}\left(f_{n}\right) \approx \mathbf{0}, l=1,2, \ldots, L_{1}, n=1,2, \ldots, N_{f}$. By blocking all possible distorted signature vectors due to different frequency offset values, $\mathbf{B}$ can effectively remove the signal component in that $\mathbf{B}^{H} \mathbf{h}_{1} \approx \mathbf{0}$. The choosing of $M^{\prime}$ is a tradeoff between the blocking effect and degree of freedom for adaptive nulling. With a fixed $\Delta F_{s}$ and $L_{1}$, a small $M^{\prime}$ leads to better blocking but poor interference suppression and vice versa. A heuristic choice which has been confirmed by numerical results is $M^{\prime} \approx M-2 L_{1}$, i.e., two degrees of freedom are used for blocking each finger. By removing both the signal and ICI components before adaptive filtering, the GSC adaptive filters can put all their efforts suppressing the MAI as desired. As a final remark, the blocking matrix in (30) can be computed in advance given a predetermined $L_{1}$ and $\Delta F_{s}$.

\section{RAKE Combining and Decisions-Aided Signal Reconstruction}

The GSC adaptive filters are a variation of the LCMV combiner and share the same characteristics of the latter, e.g., good interference cancellation and poor convergence. By poor convergence, it is meant that there is usually a certain degradation due to finite data samples. In [6], an analysis of the LCMV beamfomer reveals that the main cause of its poor convergence is the presence of a nonzero cross correlation between the signal and interference-plus-noise due to finite data samples. The cross-correlation term induces a perturbation on the beamformer weight vector, which in turn causes a drop in output SINR. With the increase of data sample size, this cross correlation gradually vanishes and the LCMV beamformer approaches the optimal MSINR beamformer. The same statements apply to GSC adaptive filters. First, the data correlation matrix in (27) is replaced by its sample average version under finite data samples

$$
\hat{\mathbf{R}}_{x}^{(j)}=\frac{1}{N_{s}} \sum_{i=1}^{N_{s}} \mathbf{x}^{(j)}(i) \mathbf{x}^{(j)}(i)=\hat{\mathbf{R}}_{s}+\hat{\mathbf{R}}_{i n}+\hat{\mathbf{R}}_{s, i n}
$$

where $\hat{\mathbf{R}}_{s}, \hat{\mathbf{R}}_{\text {in }}$, and $\hat{\mathbf{R}}_{s, \text { in }}$ are the sample signal correlation matrix, interference-plus-noise correlation matrix, and cross correlation matrix between signal and interference-plus-noise. Using the fact $\mathbf{B}^{H} \hat{\mathbf{R}}_{s} \approx 0$, we have

$$
\begin{aligned}
\mathbf{w}_{1, l}^{(j)}=\left[\mathbf{I}-\mathbf{B}\left(\mathbf{B}^{H} \hat{\mathbf{R}}_{i n} \mathbf{B}\right)^{-1} \mathbf{B}^{H} \hat{\mathbf{R}}_{i n}\right] \mathbf{c}_{1, l} \\
-\mathbf{B}\left(\mathbf{B}^{H} \hat{\mathbf{R}}_{i n} \mathbf{B}\right)^{-1} \mathbf{B}^{H} \hat{\mathbf{R}}_{s, i n} \mathbf{c}_{1, l} .
\end{aligned}
$$

Note that the first term on the righthand side of (32) represents the "optimal" MSINR weight vector in (20) [12], and the second term represents the perturbation leading to poor convergence [6]. A natural way to remedy this is then by removing the perturbation term, which can be achieved by removing the signal component in $\mathbf{x}^{(j)}(i)$ such that $\hat{\mathbf{R}}_{s, \text { in }}=\mathbf{O}$. This suggests an iterative procedure in which the signal is estimated, reconstructed at the $j$ th iteration, and subtracted from $\mathbf{x}^{(j+1)}(i)$ at the $(j+1)$ th iteration.

1) Frequency Offset Estimation and RAKE Combining: At the $j$ th iteration, the slave adaptive filters in the upper branch are used to despread the original post-FFT data $\mathbf{x}(i)$

$$
\tilde{y}_{1, l}^{(j)}(i)=\mathbf{w}_{1, l}^{(j)}{ }^{H} \mathbf{x}(i) \approx \mathbf{c}_{1, l}^{(j)}{ }^{H} \mathbf{h}_{1} d_{1, \epsilon}(i)+\mathbf{w}_{1, l}^{(j)}{ }^{H} \mathbf{n}(i)
$$

for $l=1, \ldots, L_{1}$. After despreading, the MAI is suppressed to a certain extent, and the frequency offset estimate $\hat{\epsilon}_{1}^{(j)}$ can be obtained. Methods such as the MLE described in (22) can be employed, but found to yield a large estimation variance due to residual MAI. As a more robust alternative approach, the Fourier method can be employed which starts with the $N_{t}$-point FFT of the sequence $\left\{\tilde{y}_{1, l}^{(j)}(i)\right\}$

$$
Y_{1, l}^{(j)}(k)=\operatorname{FFT}\left\{\tilde{y}_{1, l}^{(j)}(i)\left[\hat{d}_{1}^{(j-1)}(i)\right]^{*}\right\}
$$

where $\left\{\hat{d}_{1}^{(j-1)}(i)\right\}$ denote either the pilot symbols only (for $j=$ 1 ) or pilot symbols plus data decisions from the previous iteration (for $j=2,3, \ldots$ ). The FFT output power corresponding 
TABLE I

ALGORITHM SUMMARY OF PROPOSED RECEIVER

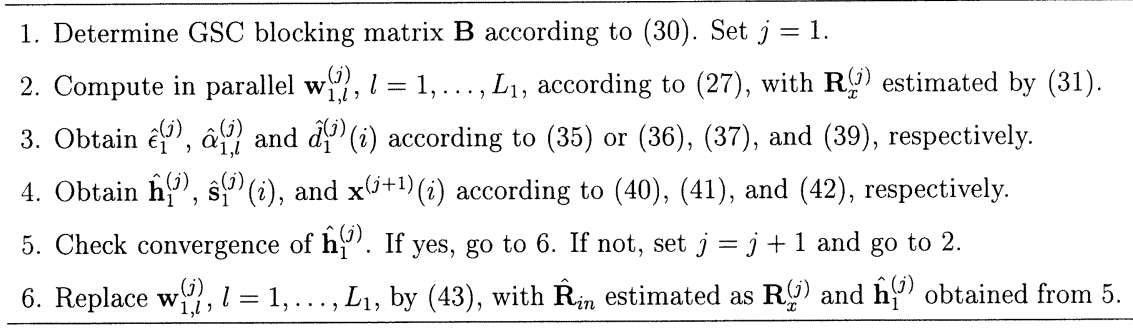

to different fingers are then incoherently summed to acquire the initial frequency offset estimate

$$
\hat{\epsilon}_{1}^{(1)}=\frac{1}{N_{t}} \arg \max _{k} \sum_{l=1}^{L_{1}}\left|Y_{1, l}^{(1)}(k)\right|^{2} .
$$

For $j \geq 2$, a coherent estimator can be constructed as

$$
\hat{\epsilon}_{1}^{(j)}=\frac{1}{N_{t}} \arg \max _{k}\left|\sum_{l=1}^{L_{1}}\left[\hat{\alpha}_{1, l}^{(j-1)}\right]^{*} Y_{1, l}^{(j)}(k)\right|^{2}
$$

where $\hat{\alpha}_{1, l}^{(j-1)}$ s are the path gain estimates from the previous iteration. Alternatively, since the MAI has been sufficiently suppressed, the MLE can be readily applied using the formula in (22), with $\hat{d}_{1, \epsilon}(i)$ replaced by the tentative RAKE combiner output $\sum_{l=1}^{L_{1}}\left[\hat{\alpha}_{1, l}^{(j-1)}\right]^{*} \tilde{y}_{1, l}^{(j)}(i)$ and $d_{1}(i)$ replaced by $\hat{d}_{1}^{(j-1)}(i)$.

Next, with $\tilde{y}_{1, l}^{(j)}(i), \hat{d}_{1}^{(j-1)}(i)$, and $\hat{\epsilon}_{1}^{(j)}$, the path gains can be estimated at the $j$ th iteration as

$$
\hat{\alpha}_{1, l}^{(j)}=\frac{1}{M N_{p}} \sum_{i=1}^{N_{p}} \tilde{y}_{1, l}^{(j)}(i)\left[\hat{d}_{1}^{(j-1)}(i)\right]^{*} e^{-j 2 \pi \hat{\epsilon}_{1}^{(j)} i}
$$

for $l=1, \ldots, L_{1}$. Based on these estimates, coherent RAKE combining is achieved by

$$
\hat{z}_{1}^{(j)}(i)=\sum_{l=1}^{L_{1}}\left[\hat{\alpha}_{1, l}^{(j)}\right]^{*} \tilde{y}_{1, l}^{(j)}(i)
$$

which is then sent to the data decision device

$$
\hat{d}_{1}^{(j)}(i)=\operatorname{dec}\left\{\hat{z}_{1}^{(j)}(i) e^{-j 2 \pi \hat{\epsilon}_{1}^{(j)} i}\right\} \text {. }
$$

It is suggested that hard decisions be used at the initialization of the iterations to avoid large errors in signal reconstruction [see (41)] due to MAI. With MAI suppressed after the first iteration, it is shown that both soft and hard decisions give similar results.

2) Channel Estimation, Signal Reconstruction, and Subtraction: As an analogy to (14), the composite channel vector estimation is accomplished by exploiting $\hat{\alpha}_{1, l}^{(j)}, \hat{\epsilon}_{1}^{(j)}$, and signal's signature vector $\left(\mathbf{c}_{1, l} \mathrm{~s}\right)$

$$
\hat{\mathbf{h}}_{1}^{(j)}=\sum_{l=1}^{L_{1}} \hat{\alpha}_{1, l}^{(j)} \mathbf{c}_{1, l}+\hat{\mathbf{v}}_{1}^{(j)}
$$

where $\hat{\mathbf{v}}_{1}^{(j)}$ is the estimated ICI vector obtained based on $\hat{\epsilon}_{1}^{(j)}$, $\hat{\alpha}_{1, l}^{(j)} \mathrm{s}, \mathbf{c}_{1, l}$ and (9) and (12). Next, with $\hat{\mathbf{h}}_{1}^{(j)}, \hat{d}_{1}^{(j)}(i)$, and $\hat{\epsilon}_{1}^{(j)}$ available, signal reconstruction can be done by

$$
\hat{\mathbf{s}}_{1}^{(j)}(i)=\hat{\mathbf{h}}_{1}^{(j)} \hat{d}_{1}^{(j)}(i) e^{j 2 \pi \hat{\epsilon}_{1}^{(j)} i} .
$$

Finally, the reconstructed signal is subtracted from the data sent to the next iteration. This leads to the residual data given by

$$
\mathbf{x}^{(j+1)}(i)=\mathbf{x}(i)-\hat{\mathbf{s}}_{1}^{(j)}(i) .
$$

By using $\mathbf{x}^{(j+1)}(i)$ as the new input of the GSC adaptive filters in (23), the adverse poor convergence can be effectively improved. This above described procedure can be iterated several times (three to four iterations for typical scenarios) to gain further improvement and approach the performance of the ideal MSINR receiver.

\section{IMPLEMENTATION AND PERFORMANCE ISSUES}

\section{A. MSINR Receiver and Algorithm Summary}

With signal subtraction, the proposed receiver will act like the optimal MSINR receiver operating on $\mathbf{R}_{i n}$. In particular, as iterations proceed, $\mathbf{x}^{(j)}(i)$ will contain only the interference and noise such that $\mathbf{R}_{x}^{(j)} \approx \hat{\mathbf{R}}_{i n}$, and $\hat{\mathbf{h}}_{1}^{(j)}$ will approach the true composite channel vector in (14). It is, thus, natural to replace the adaptive filter bank at the final iteration [with superscript $(j)$ omitted] by a single receiver with the weight vector [12]

$$
\mathbf{w}_{1}=\left[\mathbf{I}-\mathbf{B}\left(\mathbf{B}^{H} \hat{\mathbf{R}}_{i n} \mathbf{B}\right)^{-1} \mathbf{B}^{H} \hat{\mathbf{R}}_{i n}\right] \hat{\mathbf{h}}_{1} \equiv \hat{\mathbf{R}}_{i n}^{-1} \hat{\mathbf{h}}_{1}
$$

where $\mathbf{B}$ is a full rank $M \times(M-1)$ matrix satisfying $\mathbf{B}^{H} \hat{\mathbf{h}}_{1}=$ $\mathbf{0}$. It is noteworthy that the above alternative is nearly identical to the MSINR receiver and provides better MAI suppression in a heavily loaded system due to its larger degree of freedom for nulling. Nevertheless, (32) and (43) offer nearly the same performance under moderately loaded scenarios. The complete algorithm of the proposed receiver is summarized in Table I.

\section{B. PA Implementation for Multiuser Scenario}

In the direct matrix inversion (DMI) implementation, the computation of adaptive weight vector in (27) involves the inversion of $\mathbf{B}^{H} \mathbf{R}_{x}^{(j)} \mathbf{B}$, which is $M^{\prime} \times M^{\prime}$. With a large $M^{\prime}$, this would lead to a high computational load and poor convergence for real-time implementation. To alleviate this, partial adaptivity can be incorporated to reduce the size of $\mathbf{u}_{1, l}^{(j)} \mathrm{s}$ by working with a reduced size B. Here, a technique suitable for multiuser scenarios is developed. In a multiuser scenario, the MAIs composite channel vectors can be obtained by pilot symbols assisted path gain and frequency offset estimation in the same way as that described in Section II. In particular, the composite channel vector of user $k$ can be obtained by

$$
\hat{\mathbf{h}}_{k}=\sum_{l=1}^{L_{k}} \hat{\alpha}_{k, l} \mathbf{c}_{k, l}+\hat{\mathbf{v}}_{k}
$$


for $k=2,3, \ldots, K$, where $\hat{\alpha}_{k, l} \mathrm{~s}, \mathbf{c}_{k, l}$, and $\hat{\mathbf{v}}_{k}$ are defined in the same way as $\hat{\alpha}_{1, l} \mathrm{~s}, \mathbf{c}_{1, l}$, and $\hat{\mathbf{v}}_{1}$, respectively.

An essential criterion for choosing a reduced size blocking matrix is such that the upper and lower branch outputs of the GSC have a large cross correlation [13]. Since the lower branch contains no signal, the only way to maximize the cross correlation is to retain as much MAI as possible in the lower branch. This suggests that a suitable method for implementing the PA receiver is to find a reduced size $\mathbf{B}$ that can retain as much MAI as possible. By doing so, a mutual cancellation of MAI can be achieved between the upper and lower branches. With $\hat{\mathbf{h}}_{k}$ s available, a reduced size blocking matrix $\overline{\mathbf{B}}$ can be obtained by projecting onto the column space of $\mathbf{B}$ these set of vectors

$$
\overline{\mathbf{B}}=\mathbf{B B}^{H}\left[\hat{\mathbf{h}}_{2}, \hat{\mathbf{h}}_{3}, \ldots, \hat{\mathbf{h}}_{K}\right]
$$

where we have assumed that the columns of $\mathbf{B}$ form an orthonormal set. PA realization via (45) is simple and proves robust to errors in MAIs channel estimates. In particular, errors in $\hat{\mathbf{h}}_{k} \mathrm{~s}$ tend to decrease the cross correlation between the two branches, and results in only slight performance degradation. When viewed as a multiuser detector, the proposed PA receiver is much more robust than the conventional ones, which detect and subtract the MAI [7]. In conventional multiuser detectors, a phase error in MAIs channel estimate can result in an enhanced MAI power and possible error propagation.

\section{Recursive Computation of Weight Vectors}

For a more efficient implementation, the GSC weight vector and path gain estimation can be done in a time-recursive fashion using stochastic gradient algorithms such as LMS [14]. For the computation of GSC weight vector in (27), we have the recursive formulation

$$
\begin{aligned}
\mathbf{u}_{1, l}^{(j)}(i+1)= & \mathbf{u}_{1, l}^{(j)}(i)+\mu_{u}\left[\mathbf{c}_{1, l}^{H} \mathbf{x}^{(j)}(i)-\mathbf{u}_{1, l}^{(j)} \mathbf{B}^{H} \mathbf{x}^{(j)}(i)\right]^{*} \\
& \times \mathbf{B}^{H} \mathbf{x}^{(j)}(i) \\
\mathbf{w}_{1, l}^{(j)}(i+1)= & \mathbf{c}_{1, l}-\mathbf{B} \mathbf{u}_{1, l}^{(j)}(i+1)
\end{aligned}
$$

for $i=1,2, \ldots$ On the other hand, a recursive algorithm for the estimation of path gain $\hat{\alpha}_{1, l}^{(j)}$ in (37) is given by [15]

$$
\begin{aligned}
& \hat{\alpha}_{1, l}^{(j)}(i+1)=\hat{\alpha}_{1, l}^{(j)}(i) \\
& \quad+\mu_{a}\left[\frac{1}{M} \tilde{y}_{1, l}^{(j)}(i) e^{-j 2 \pi \hat{\epsilon}_{1}^{(j)} i}-\hat{\alpha}_{1, l}^{(j)}(i) \hat{d}_{1}^{(j-1)}(i)\right]\left[\hat{d}_{1}^{(j-1)}(i)\right]^{*} .
\end{aligned}
$$

In the above, $\mu_{u}$ and $\mu_{a}$ are the adaptation stepsizes.

\section{COMPUTER Simulations}

Simulation results are demonstrated to confirm the performance of the proposed receiver in a time-multiplexed pilot symbols assisted system. For all users, $L_{k}=3$ independent Rayleigh-fading paths were generated with the delays $\tau_{k, l} \mathrm{~s}$ chosen from $\left\{0, T_{c}, 2 T_{c}\right\}$, which was smaller than the guard interval $T_{G}=8 T_{c}$. The path gains $\bar{\alpha}_{k, l} \mathrm{~s}$ were assumed i.i.d. unit variance complex Gaussian random variables. The number of subcarriers was chosen to be $M=32$ equal to the processing gain. All MC-CDMA signals were generated

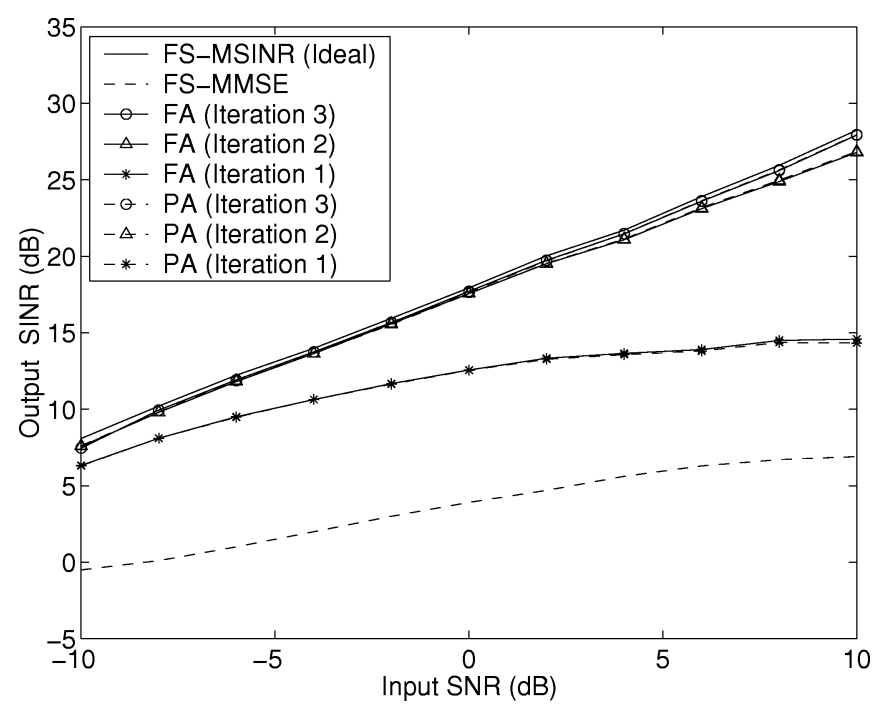

Fig. 4. Output SINR versus input SNR, with MSR $=10 \mathrm{~dB}, N_{s}=300$, $K=10$, and $\epsilon_{1}=0.2$.

with binary phase-shift keying (BPSK) data modulation and orthogonal gold codes were used as the spreading codes. Also, the frequency offsets of all users were assumed to be in the range $\Delta F_{s}=\left[-T_{b} / 2, T_{b} / 2\right]$ such that $-0.5 \leq \epsilon_{k} \leq 0.5$. The blocking matrix $\mathbf{B}$ was constructed by the eigenvector method described in Section III-B, with $N_{f}=50$ and $M^{\prime}=26$. The length of FFT in (34) was chosen to be $N_{t}=1024$, with zero padding applied. As a performance index, the output SINR is defined to be the ratio of the signal power $\left(\left|\mathbf{w}_{1}^{H} \mathbf{h}_{1}\right|^{2}\right)$ to MAI-plus-noise power $\left(\sum_{k=2}^{K}\left|\mathbf{w}_{1}^{H} \mathbf{h}_{k}\right|^{2}+\sigma_{n}^{2} \mathbf{w}_{1}^{H} \mathbf{w}_{1}\right)$ at the receiver output. Also, the input SNR was defined as $\mathrm{SNR}_{i}=\sigma_{1}^{2} / \sigma_{n}^{2}$, and the MAI-to-signal ratio (MSR) was defined as MSR $=\sigma_{k}^{2} / \sigma_{1}^{2}, k=2, \ldots, K$, where we assumed equal power MAI. For each simulation trial, $N_{s}$ symbols (including data and pilot) were used to obtain the sample estimate of $\mathbf{R}_{x}^{(j)}$, and $N_{p}$ pilot symbols were used to obtain $\hat{\epsilon}_{1}^{(j)}$ and $\hat{\alpha}_{1, l}^{(j)} \mathrm{s}$ at the first iteration. A total of 500 Monte Carlo trials were executed to obtain one output SINR value, with each trial using a different set of $\bar{\alpha}_{k, l} \mathrm{~s}$ and data/noise sequence. For performance comparison, the results obtained with the ideal FS-MSINR receiver and FS-MMSE receiver were also included. The ideal FS-MSINR receiver was implemented by artificially removing the signal component in the data, and using the true composite channel vector $\mathbf{h}_{1}$ to obtain (20). On the other hand, the FS-MMSE receiver was implemented by first estimating the frequency offset using (35) with directly despread data, followed by composite channel vector estimation to obtain (17). Finally, the following "standard" parameters will be used throughout the section unless otherwise mentioned: $\mathrm{SNR}_{i}=0 \mathrm{~dB}, \mathrm{MSR}=10 \mathrm{~dB}, N_{s}=300, K=10, \epsilon_{1}=0.2$, $N_{p} / N_{s}=1 / 10$. Except for one case, the true MAI channel vectors were assumed in (45), i.e., $\hat{\mathbf{h}}_{k}=\mathbf{h}_{k}, k=2, \ldots, K$.

In the first set of simulations, the output SINR performance is evaluated as a function of input SNR for both the fully adaptive (FA) and PA versions of the proposed receiver. The results shown in Fig. 4 indicate that the proposed receiver successively approaches the ideal MSINR receiver, with a degradation of only about $0.2 \mathrm{~dB}$ in three iterations. The FS-MMSE receiver performs poorly due to poor frequency offset estimation prior 


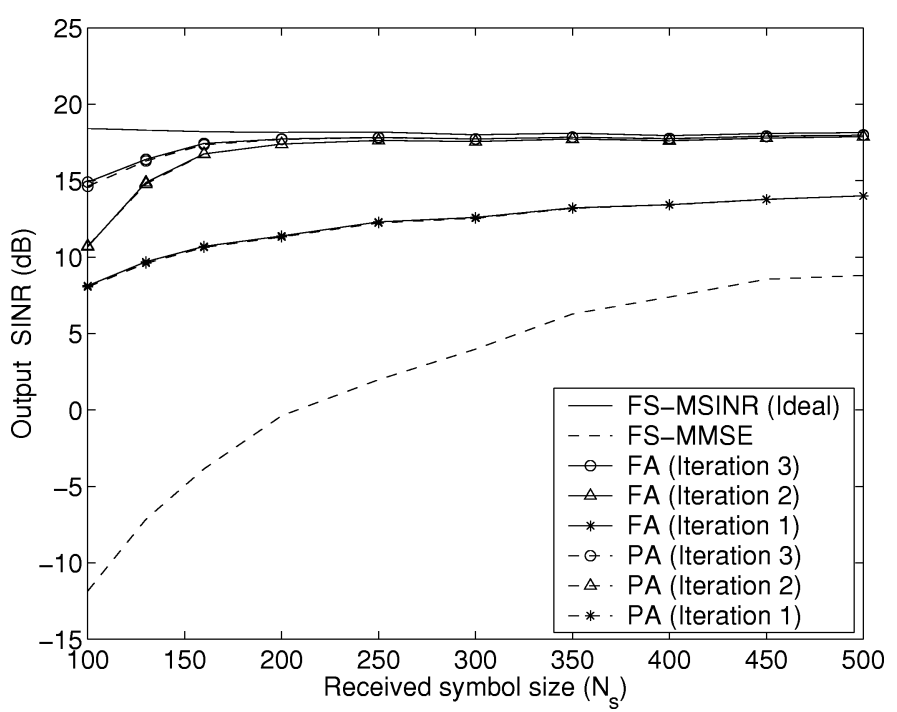

Fig. 5. Output SINR versus received symbol size $N_{s}$, with $\mathrm{SNR}_{i}=0 \mathrm{~dB}$, $\mathrm{MSR}=10 \mathrm{~dB}, K=10$, and $\epsilon_{1}=0.2$.

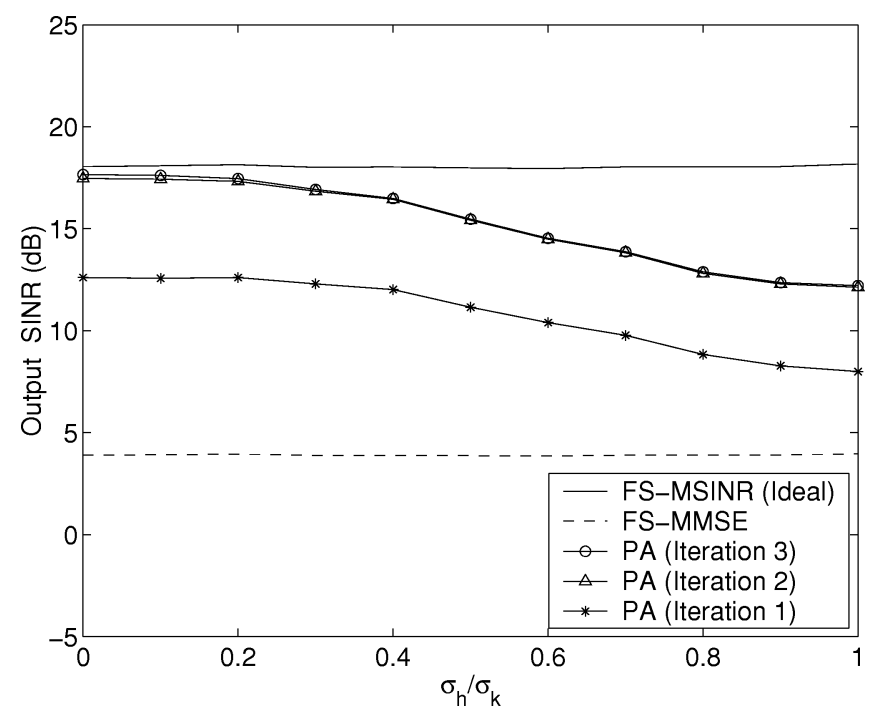

Fig. 6. Output SINR versus relative channel estimation error $\sigma_{h} / \sigma_{k}$, with $\mathrm{SNR}_{i}=0 \mathrm{~dB}, \mathrm{MSR}=10 \mathrm{~dB}, N_{s}=300, K=10$, and $\epsilon_{1}=0.2$.

to MAI cancellation. On the other hand, the PA receiver has almost the same performance of the FA receiver, confirming the assertion in Section IV-B. In the second set of simulations, the output SINR performance is evaluated as a function of received symbol size $N_{s}$. The results given in Fig. 5 show that the proposed receiver successively improves as iterations proceed, approaching the ideal MSINR receiver within about 300 symbols. These results confirm that the MAIs were indeed successfully suppressed by the proposed receiver, and PA implementation can retain the performance of the FA receiver. In the following, only the proposed PA and FS-MMSE receivers will be evaluated.

In the third set of simulations, the robustness of the proposed PA receiver against MAI channel estimation errors is demonstrated. In this case, $\hat{\mathbf{h}}_{k}=\mathbf{h}_{k}+\sigma_{h} \Delta \mathbf{h}$ in (45), where $\Delta \mathbf{h}$ is a random vector with the entries being i.i.d. complex Gaussian random variables with the same variance of 3 . Note that the entries of $\mathbf{h}_{k}$ are i.i.d. complex Gaussian random variables with variance $L_{k} \sigma_{k}^{2}=3 \sigma_{k}^{2}$. Fig. 6 shows the output SINR versus

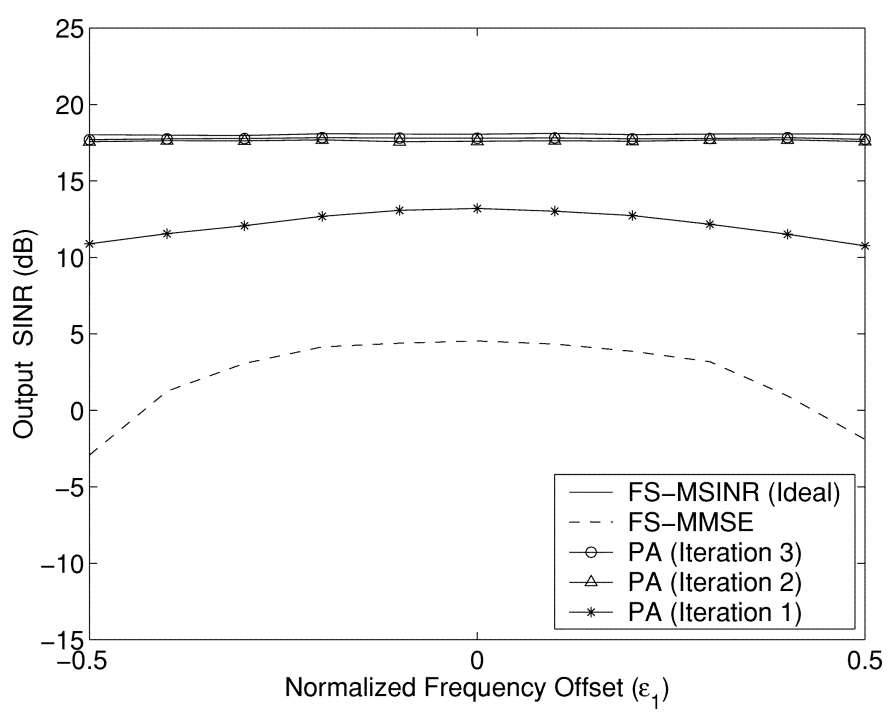

Fig. 7. Output SINR versus normalized frequency offset $\epsilon_{1}$, with $\mathrm{SNR}_{i}=$ $0 \mathrm{~dB}, \mathrm{MSR}=10 \mathrm{~dB}, N_{s}=300$, and $K=10$.

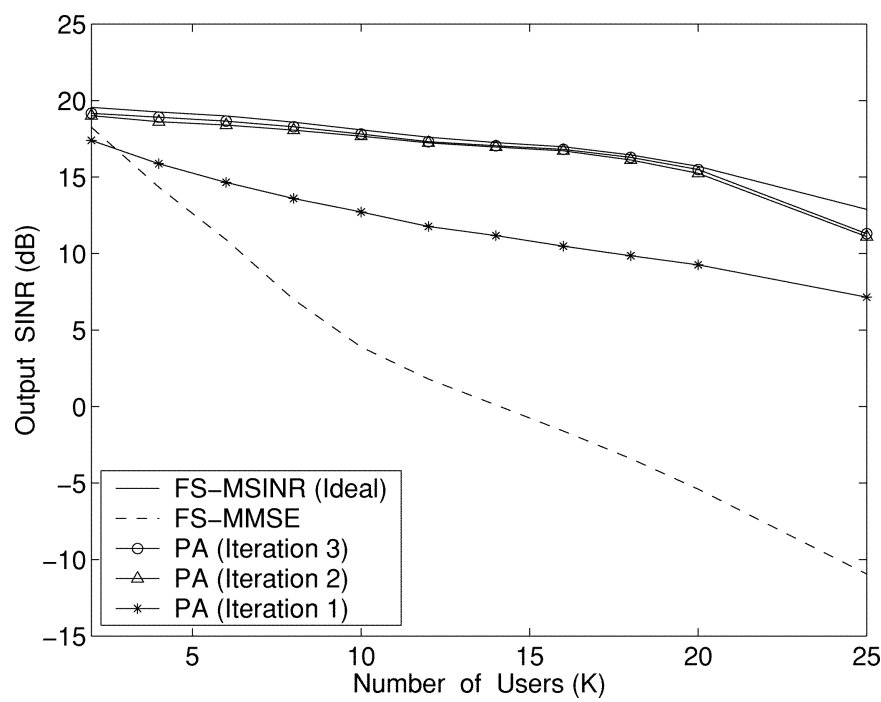

Fig. 8. Output SINR versus user number $K$, with $\mathrm{SNR}_{i}=0 \mathrm{~dB}$, MSR $=10 \mathrm{~dB}, N_{s}=300$, and $\epsilon_{1}=0.2$.

$\sigma_{h} / \sigma_{k}$. The results confirm that the proposed PA receiver can perform quite reliably for up to a $20 \%$ relative error in MAI channel estimation. In the fourth set of simulations, the tolerance of the proposed PA receiver against different frequency offsets is evaluated. The resulting output SINR curves as a function of $\epsilon_{1}$ are plotted in Fig. 7, which shows that within the entire range $-0.5 \leq \epsilon_{k} \leq 0.5$, the output SINR is almost constant. The results indicate that the modified blocking matrix $\mathbf{B}$ effectively removes the signal and ICI together so as to avoid possible signal cancellation in the GSC adaptive filters. With successful signal reception and MAI suppression by the adaptive filters, an accurate frequency offset estimate can then be obtained to guarantee a high output SINR.

In the fifth set of simulations, the system capacity is evaluated with different values of $K$. As shown in Fig. 8, the proposed receiver again successively approaches the MSINR receiver, with a degradation of about $2 \mathrm{~dB}$ in output SINR with $K=25$. The simulation results confirm that the proposed receiver is able to offer the performance of the optimal MSINR receiver with a 


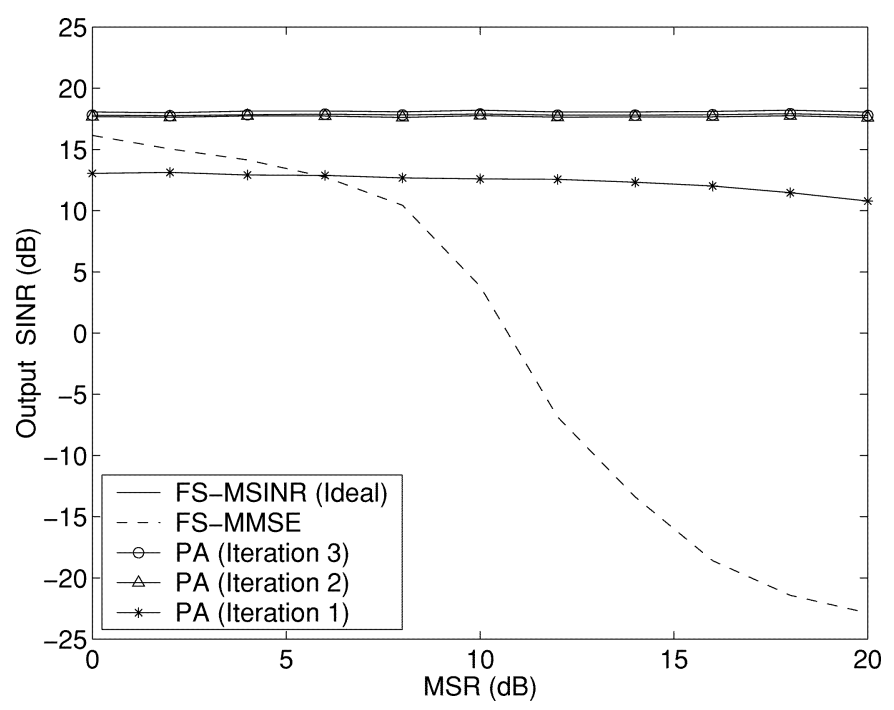

Fig. 9. Output SINR versus MSR, with $\mathrm{SNR}_{i}=0 \mathrm{~dB}, N_{s}=300, K=10$, and $\epsilon_{1}=0.2$.

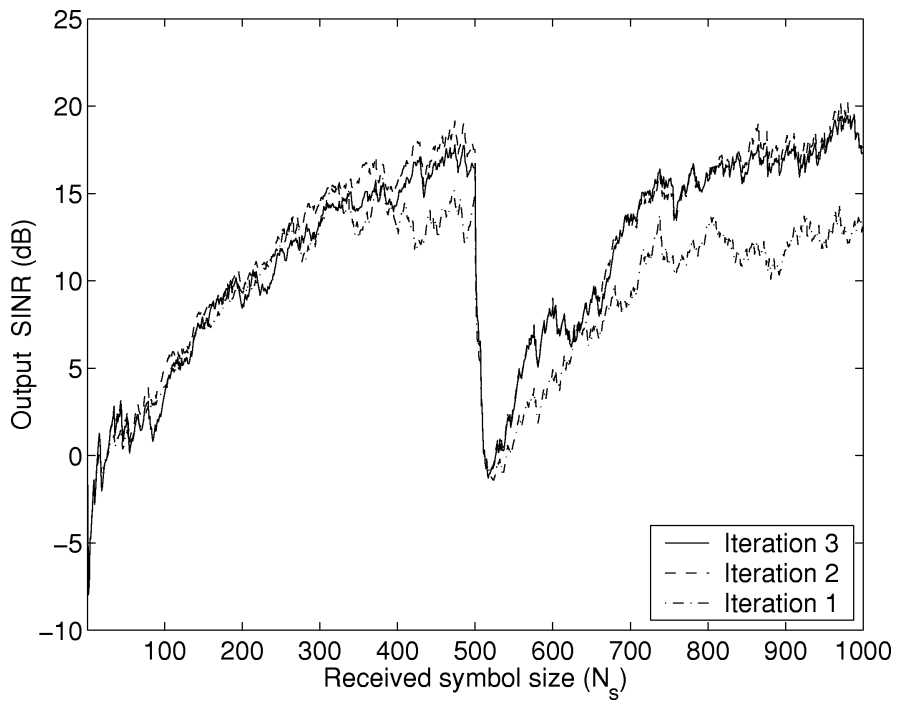

Fig. 10. Evaluation of recursive algorithms with $\mathrm{SNR}_{i}=0 \mathrm{~dB}$, MSR $=10 \mathrm{~dB}, K=5$, and $\epsilon_{1}=0.2$. Multipath gains changed at 500th symbol.

moderate symbol size in a heavily loaded system. In the sixth set of simulations, the near-far resistance of the proposed receiver is evaluated with different MSR values. Fig. 9 shows the output SINR curves. It is observed that the proposed receiver achieves its excellent near-far resistance by successfully cancelling the strong MAI using the temporal degree of freedom offered by the predespread data.

Finally, to demonstrate the effectiveness of the recursive algorithms for weight vector adaptation, we replaced the direct weight vector computation in (27) and path gain estimation in (37) by the formulae given in (46) and (47), respectively. In this case, the FA receiver was used, with $K=5$ and adaptation stepsizes chosen as $\mu_{u}=10^{-5}$ and $\mu_{v}=0.05$, respectively. In order to demonstrate the tracking capability of these algorithms, the multipath fading gains of all users were deliberately changed at the 500th symbol. The resulting learning curves shown in Fig. 10 show that the proposed receiver con- verges in about 300 symbols, and the recursive algorithms successfully adjusted their weights to adapt to the environmental changes.

\section{CONCLUSION}

A robust iterative MC-CDMA receiver with adaptive MAI suppression has been proposed for a pilot symbols assisted system over a multipath fading channel with frequency offset. The receiver consists of two branches. In the lower branch, a "master" adaptive filter bank based on the GSC technique is constructed to perform effective suppression of MAI. In order to enhance the convergence behavior of the GSC, a decisions-aided scheme is proposed, in which the signal waveform is first reconstructed and then subtracted from the input data sent to the next iteration. In the upper branch, a "slave" adaptive filter bank, copied from the lower branch, performs despreading and MAI suppression, and pilot symbols assisted frequency offset estimation, channel vector estimation and RAKE combining give the desired signal symbols. With signal subtraction in the lower branch, the proposed MC-CDMA receiver can achieve nearly the performance of the ideal MSINR receiver within a few iterations. Finally, a low-complexity PA realization of the GSC adaptive filters is presented for a multiuser scenario. The new PA receiver is shown to be robust to multiuser channel errors, and offer nearly the same performance of the fully adaptive receiver. In summary, the proposed MC-CDMA receiver with PA MAI suppression performs near optimal signal detection with tolerance to large frequency offsets and resistance to strong MAI. More importantly, it can be initialized in the blind mode without the aid of channel estimation and frequency offset compensation.

\section{REFERENCES}

[1] S. Hara and R. Prasad, "Overview of multicarrier CDMA," IEEE Commun. Mag., vol. 35, pp. 126-133, Dec. 1997.

[2] G. Woodward and B. S. Vucetic, "Adaptive detection for DS-CDMA," Proc. IEEE, vol. 86, pp. 1413-1434, July 1998.

[3] P. H. Moose, "A technique for orthogonal frequency division multiplexing frequency offset correction," IEEE Trans. Commun., vol. 42, pp. 2908-2914, Oct. 1994.

[4] B. D. Van Veen and K. M. Buckley, "Beamforming: A versatile approach to spatial filtering," IEEE ASSP Mag., vol. 5, pp. 4-24, Apr. 1988.

[5] T. M. Lok, T. F. Wong, and J. S. Lehnert, "Blind adaptive signal reception for MC-CDMA systems in Rayleigh-fading channels," IEEE Trans. Commun., vol. 47, pp. 464-471, Mar. 1999.

[6] M. Wax and Y. Anu, "Performance analysis of the minimum variance beamformer," IEEE Trans. Signal Processing, vol. 44, pp. 928-937, Apr. 1996.

[7] S. Moshavi, "Multiuser detection for DS-CDMA communications," IEEE Commun. Mag., vol. 34, pp. 124-136, Oct. 1996.

[8] J. S. Goldstein and I. S. Reed, "Subspace selection for partially adaptive sensor array processing," IEEE Trans. Aerosp. Electron. Syst., vol. 33, pp. 539-544, Apr. 1997.

[9] R. Van Nee and R. Prasad, OFDM Wireless Multimedia Communications. London: Artech House, 2000.

[10] T. M. Schmidl and D. C. Cox, "Robust frequency and timing synchronization for OFDM," IEEE Trans. Commun., vol. 45, pp. 1613-1621, Dec. 1997.

[11] B. Widrow, K. M. Duvall, R. P. Gooch, and W. C. Newman, "Signal cancellation phenomena in adaptive antennas: Causes and cures," IEEE Trans. Antennas Propagat., vol. AP-30, pp. 469-478, May 1982.

[12] D. H. Johnson and D. E. Dudgeon, Array Signal Processing: Concepts and Techniques. Englewood Cliffs, NJ: Prentice-Hall, 1993, ch. 7. 
[13] D. A. Pados and S. N. Batalama, "Low-complexity blind detection of DS/CDMA signals auxiliary-vector receivers," IEEE Trans. Commun., vol. 45, pp. 1586-1594, Dec. 1997.

[14] S. Haykin, Adaptive Filter Theory, 3rd ed. Englewood Cliffs, NJ: Prentice-Hall, 1996.

[15] V. G. Moghadam and M. Kaveh, "A CDMA interference canceling receiver with an adaptive blind array," IEEE J. Select. Areas Commun., vol. 16, pp. 1542-1554, Oct. 1998.

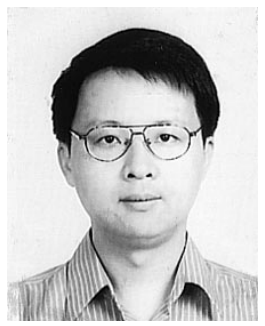

Juinn-Horng Deng (S'01) was born in Miaoli, Taiwan, R.O.C., in 1965. He received the B.S.E.E. degree from Chung Cheng Institute of Technology, Taiwan, R.O.C., in 1988, and the M.S.E.E. degree from Yuan Ze University, Taiwan, R.O.C., in 1993. Currently, he is working toward the Ph.D. degree in the Department of Communication Engineering, National Chiao Tung University, Hsinchu, Taiwan, R.O.C.

From 1988 to 1991, and 1993 to 1999, he was in the Phase Array Radar Department, Chung Shan Institute of Science and Technology, Taiwan, R.O.C. His research interests include multiuser detection, communication signal processing, and smart antennas.

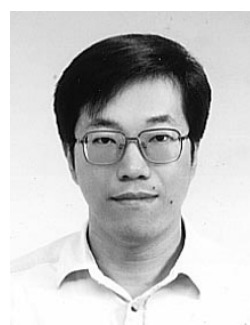

Ta-Sung Lee (S'88-M'89) was born in Taipei, Taiwan, R.O.C., in 1960. He received the B.S. degree from National Taiwan University, Taipei, Taiwan, R.O.C., the M.S. degree from the University of Wisconsin, Madison, and the Ph.D. degree from Purdue University, West Lafayette, IN, in 1983, 1987, and 1989 , respectively, all in electrical engineering.

In 1990, he joined the Faculty of National Chiao Tung University (NCTU), Taipei, Taiwan, where he holds a position as Professor in the Department of Communication Engineering. From 1999 to 2001, he was Director of the Communications and Computer Training Program at NCTU. $\mathrm{He}$ is active in research and development in advanced techniques for wireless communications, such as smart antennas for mobile cellular, BWA and WLAN systems, space-time and MIMO transceivers for high data rate transmission, OFDM based broadband wireless access systems, and interference rejection for co-existing systems. He has been involved in several National Research Programs, such as the "Program for Promoting Academic Excellence of Universities" supported jointly by the Ministry of Education and National Science Council (NSC) of R.O.C., and the program of "Advanced B3G Radio Access Technologies" supported by the National Telecommunications Program Office.

Dr. Lee is a Member of Phi Tau Phi Society of R.O.C., and recipient of the 1999 Young Electrical Engineer Award of the Chinese Institute of Electrical Engineers and the 2001 NCTU Teaching Award. 